\title{
Studies of thin-walled parts deformation by grip- ping force during turning process on an example of bearing ring
}

\author{
Adam Patalas ${ }^{1, *}$, Michat Regus ${ }^{1}$, Katarzyna Peta $^{1}$ \\ ${ }^{1}$ Poznań University of Technology, Faculty of Mechanical Engineering and Management, Piotrowo 3, \\ 60-965 Poznań, adam.patalas@put.poznan.pl; michal.j.regus@doctorate.put.poznan.pl
}

\begin{abstract}
In this paper thin-walled part deformation during finishing turning process caused by gripping force of hydraulic lathe chuck was investigated. Bearing ring was taken as an example of thin-walled part undergo finishing turning operation. Finite Element Method (FEM) was used to define the deformation of examined part. The aim of presented research was to compare the deformation of bearing ring caused by gripping force of hydraulic 3-jaw chuck and 6-jaw chuck for different values of total gripping force. The data obtained from conducted simulations allowed to evaluate the influence of gripping force on machining part deformation which is directly related with its geometrical accuracy.
\end{abstract}

Keywords: FEM, thin-walled parts, gripping force, machining part deformation

\section{Introduction}

Machining is one of the most common methods for producing parts for different branches of industry. Nevertheless, there is a group of parts, which production is associated with high technological difficulties. Thin-walled parts, which are highly susceptible for deformation, can be counted in among these parts. The following factors leads to deformation of thinwalled parts during machining: gripping force, cutting force and heat generated during process $[1,2]$. In this paper bearing ring deformation, during finishing turning of inner diameter, caused by gripping force of hydraulic chuck was investigated. In this purpose Finite Element Method was used. The drawing of analysed bearing ring is presented in fig. 1.

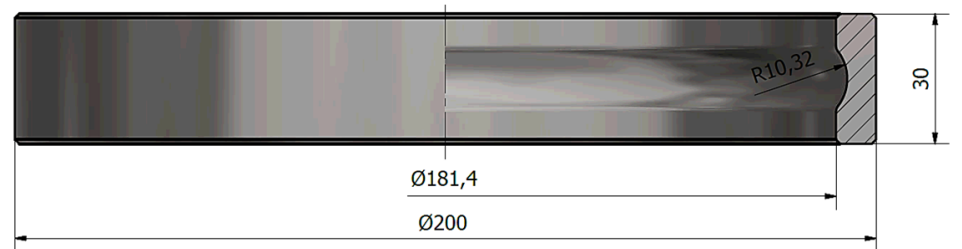

Fig. 1. Simplified drawing of thin-walled bearing ring

\footnotetext{
* Corresponding author: adam.patalas@put.poznan.pl

Reviewers: Jana Petrů,, Alžbeta Spietová
} 
A couple of research regarding deformation of thin-walled parts which undergo machining process have been already conducted. In papers [2- 5] behaviour of thin-walled workpiece during milling operation was investigated. In researches $[1,5,6]$ deformation of rings under turning process were analysed. R. Thakore et al. describes in [7] potential problems during grinding operation of bearing rings.

In this paper numerical simulations of bearing ring deformation induced by gripping force were conducted for 3-jaw chuck and 6-jaw chuck (Fig. 2).

a)

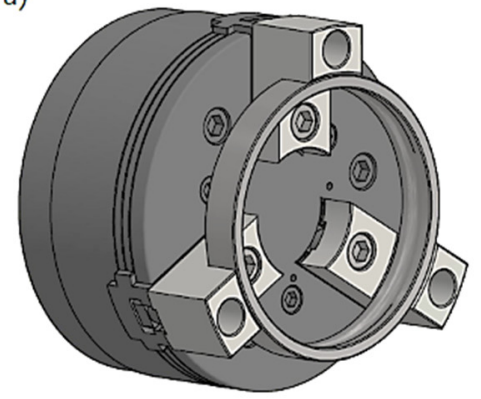

b)

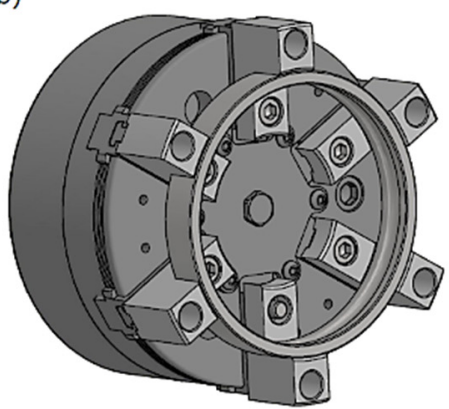

Fig. 2. Clamping strategy; a) 3-jaw chuck SCHUNK ROTA NCD 255 b) 6-jaw chuck ROTA NCR 250

Most of modern CNC lathes are equipped with hydraulic cylinder which control the opening of lathe chuck. Depends on the application, hollow and partially-hollow cylinders are in use. The principle of operation for clamping mechanism on CNC lathe is presented in fig. 3 .

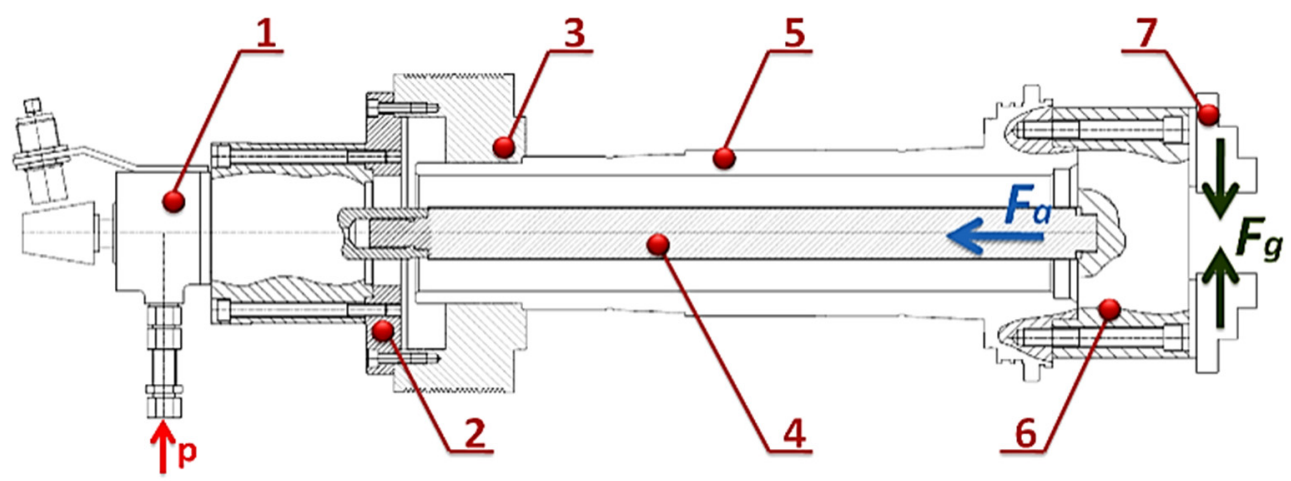

Fig. 3. Schematic drawing of clamping mechanism on $\mathrm{CNC}$ lathe; 1) Rotating hydraulic clamping cylinder, 2) Adapter of clamping cylinder, 3) Pulley of main drive, 4) Draw bar, 5) Spindle, 6) Chuck, 7) Jaws; Fa- actuating force of clamping cylinder, Fg - chuck gripping force.

For further analysis partially-clamping cylinder SMW SIN-S 100 was assumed. The operating range of clamping cylinder were limited to $7 \div 40$ bar. Active piston area of selected cylinder is $66 \mathrm{~cm}^{2}$ [8]. The theoretical characteristic of cylinder SIN-S100 is shown in fig. 4. 


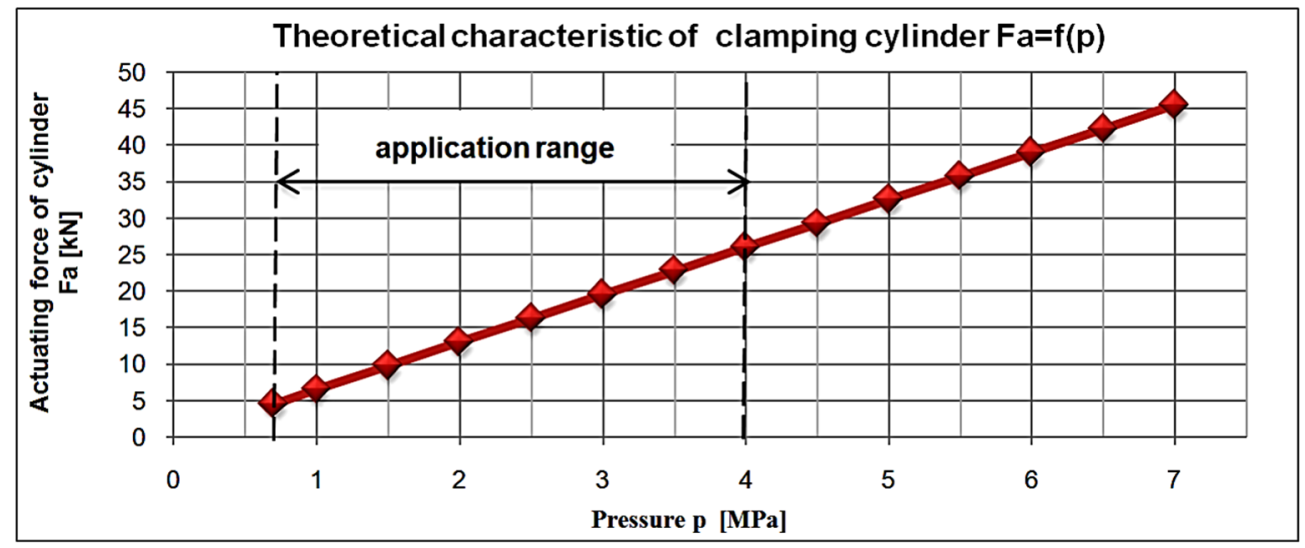

Fig. 4. Theoretical characteristic of hydraulic clamping cylinder SMW SIN-S 100 [8]

Machining process of thin-walled parts require possibly low gripping force to minimize the deformation and keep geometrical tolerances of produced parts [6]. Number of chuck's jaws plays significant role in thin-walled part deformation. The simulations of bearing ring deformation were conducted for 3-jaw chuck (ROTA NCD 255) and 6-jaw chuck (ROTA NCR 250) [8]. The characteristics of chuck total gripping force in function of pressure $[F g=f(p)]$ are presented in fig. 5 .

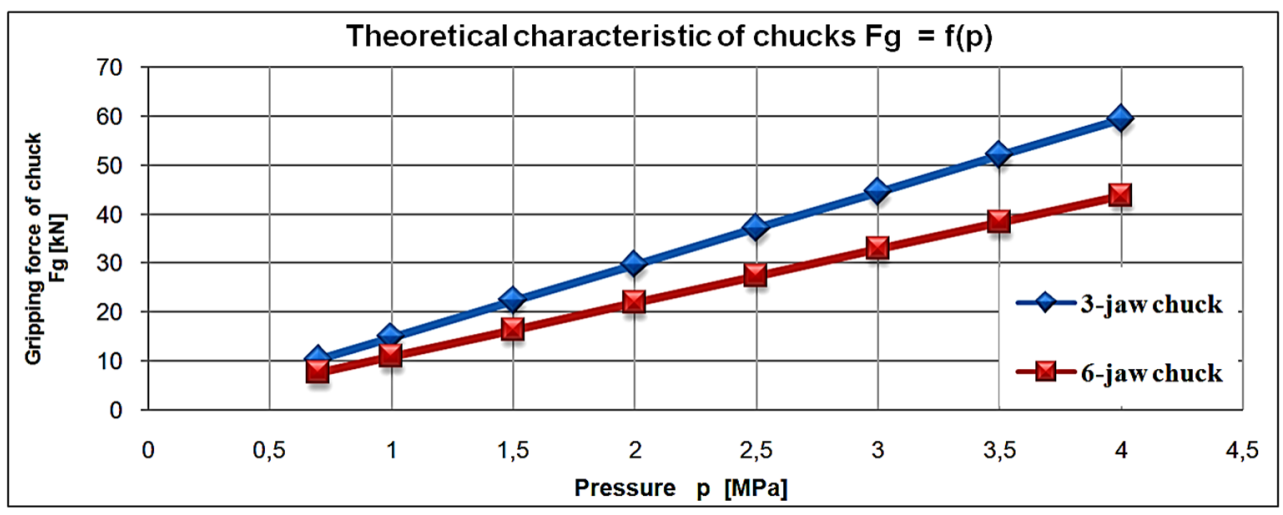

Fig. 5. Theoretical characteristics of chucks in combination with clamping cylinder SIN-S 100 [9]

\section{Methodology of research}

To evaluate the deformation of bearing ring under finishing turning conditions, Finite Element method (FEM) was applied. ANSYS software was used for simulation. Direct solver with force convergence control was taken. Augmented Lagrange method was applied for contact problem formulation. Due to the contact stress occurrence between the jaws and workpiece, static structural simulation is considered as non-linear. As simulation aims to estimate deformation of machined part, refined mesh was applied only for analysed bearing ring (Fig. 6). 
a)

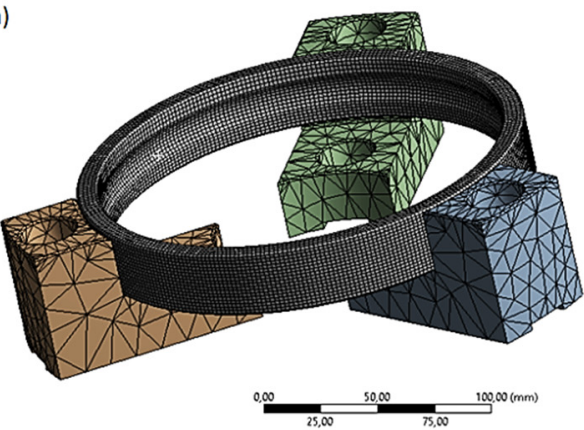

b)

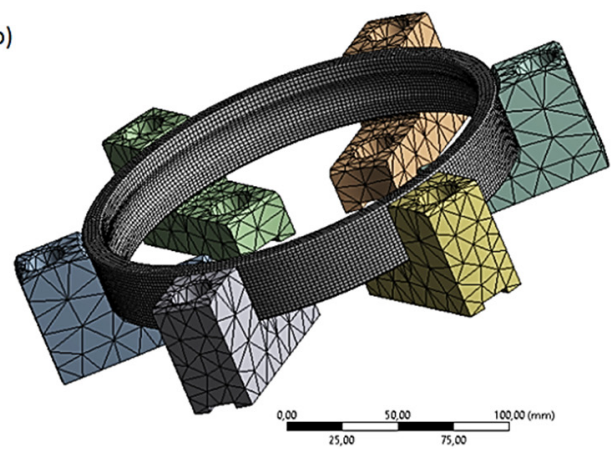

Fig. 6. Meshing problem a) 3-jaw chuck, b) 6-jaw chuck.

As a consequence of the above, for jaws tetrahedron finite element was assumed, while for workpiece hex-dominant type was chosen.

Further assumptions were taken for the simulation:

- linear model of material of bearing ring (alloy steel 100Cr6),

- linear characteristic of hydraulic clamping cylinder,

- linear characteristic of clamping chucks,

- equal clamping force at every chuck's jaw,

- inertial force working on jaws wasn't taken into consideration due to low spindle speed during turning operation at internal diameter of bearing ring (assuming cutting speed $v_{c}=240 \mathrm{~m} \cdot \mathrm{min}^{-1}$, spindle speed would be $n=421 \mathrm{rpm}$ ),

- cutting force influence was ignored during the simulation, due to low value under finishing turning condition, as well as the fact, it works steadily at the circumference of ring,

- thermal deformation during machining process was ignored in simulation, since it affects evenly the shape of ring.

\section{Results}

Base on the assumptions, presented above, simulations were conducted, to evaluate the internal strains and deformation of the bearing ring. Results of FEM analysis for the lowest gripping force are presented in figure 7. As seen in figure 7 application of 6-jaw chuck allows to reduce significantly the part deformation, and as a consequence, obtain higher roundness accuracy.

a)

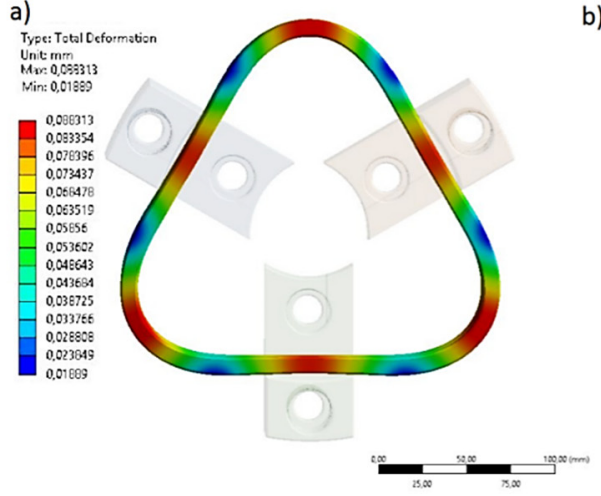

b)

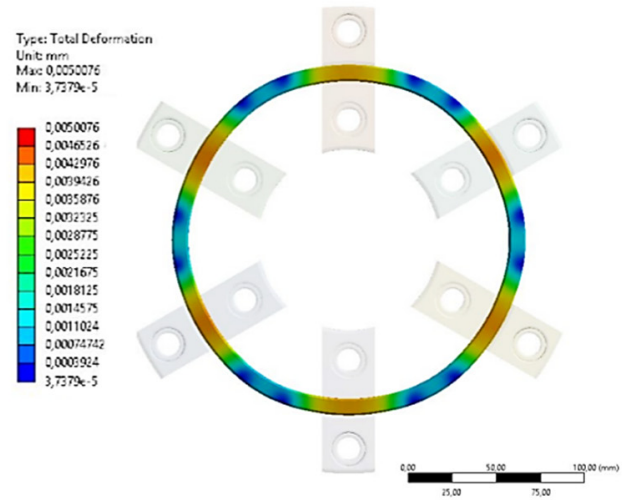


c)

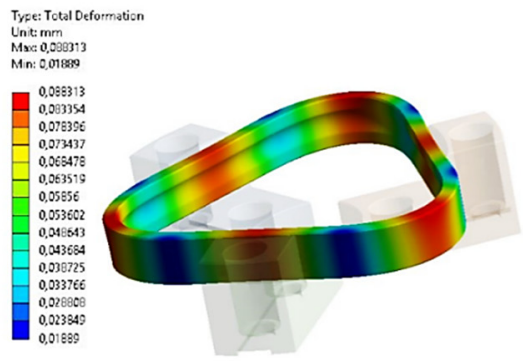

d)

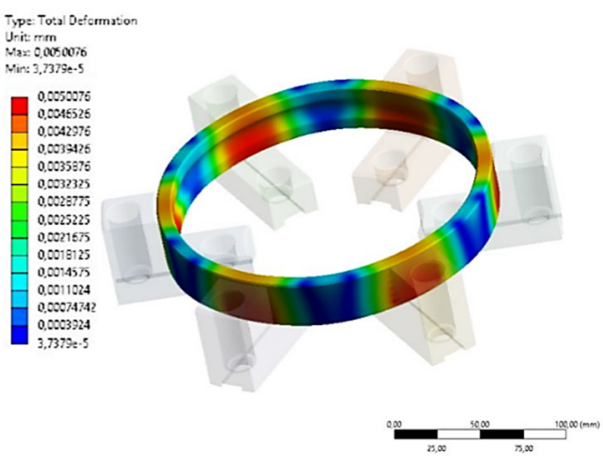

Fig. 7. Results of FEM analysis for total gripping force of lathe chuck $F g=10 \mathrm{kN}$ a),c) clamping in 3-jaw chuck with visible triangular roundness error of ring profile, b),d) clamping in 6jaw chuck with less deformation and profile error

Deformations of jaws were omitted, as this problem, however critical for overall fixture performance, was specified as non-essential in this very case. Deformations on diagrams for 3-jaw fixture are up scaled 20 times, while deformations on diagrams for 6-jaw fixture are up scaled 200 times, for better clarity of visualization and in order to emphasis characteristics. Complete results of conducted simulations for different value of gripping force are presented on below diagrams (Figure 8). As it was mentioned in previous part of this paper, the linear model of the material has been assumed. Consequently, the obtained characteristics of stress and deformation are linear. It needs to be highlighted that those results are reliable within Hooke's law application range. It's marked on the graphs below.

a)

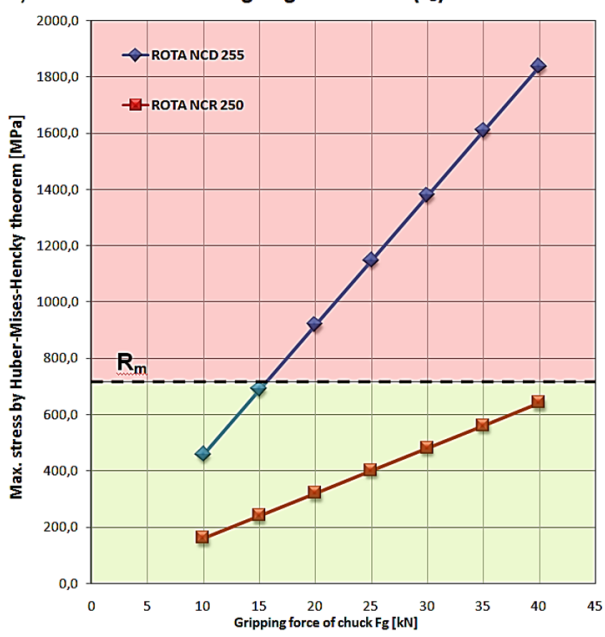

b)

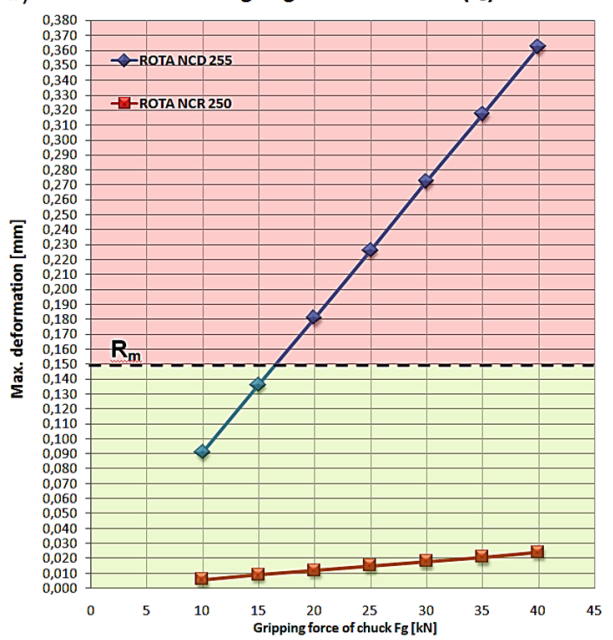

Fig. 8. Results of FEM analysis a) stress of bearing ring as a function of gripping force $\sigma=f(F g)$, b) deformation of bearing ring as a function of gripping force $\varepsilon=f(F g)$; area under dashed line represents the field of Hooke's law application

Above this range, yield limit for selected steel is exceeded, therefore linear model of material cannot be considered anymore. To evaluate plastic deformation of bearing ring nonlinear model would have to be implemented. However, it's worth to mention, that such 
simplification is reasonable in this particular case. One of the major assumption of conducted analysis, is that workpiece can undergo only elastic deformation. Exceeding the yield limit results in destruction of bearing ring, what obviously is not permissible in production process.

\section{Conclusions}

In this paper thin-walled part deformation under finishing turning condition was investigated. As a research object, bearing ring made of alloy steel was taken. To evaluate the influence of gripping force on part deformation FEM analysis was conducted. Base on the obtained results, it can be stated that application of 6-jaw chuck allows to reduce significantly the stress and deformation of thin-walled parts. According to the Figure 8, if we apply the gripping force greater than $15 \mathrm{kN}$ on 3-jaw chuck, yield limit would be exceeded. Consequently, bearing ring would be damaged. Even the lowest gripping force applied to the 3-jaw chuck $(10 \mathrm{kN})$, results in bearing ring deformation of $0.09 \mathrm{~mm}$ - which is not acceptable. With the same gripping force applied by 6-jaw chuck we can reduce the maximal deformation of bearing ring to $5 \mu \mathrm{m}$. As a result, geometrical tolerances can be fulfilled.

Base on presented mathematical model it's possible to estimate the thin-walled part deformation under gripping force of lathe chuck. Consequently, right value of hydraulic pressure can be adjusted to reach suitable gripping force, that would provide required geometrical accuracy during production process. Furthermore, presented research can be helpful for selection of appropriate $\mathrm{CNC}$ lathe equipment, like hydraulic clamping cylinder and power chuck

\section{Acknowledgement}

This research was supported by Poznań University of Technology as part of statutory research on Faculty of Mechanical Engineering and Management.

\section{References}

1. M. Estremsa, M. Arizmendia, A. J. Zabaletaa, A. Gil, Numerical Method to Calculate the Deformation of Thin Rings during Turning Operation and its Influence on the Roundness Tolerance. Procedia Engineering, 132, 872 - 879 (2015)

2. J. Loehea, M. F. Zaeha, O. Roescha, In-Process Deformation Measurement of thinwalled Workpieces. Procedia CIRP, 1, 546 - 551 (2012)

3. J. Shia, J.Gaoa, Q.Songa, Z.Liua, Y.Wana, Dynamic deformation of thin-walled plate with variable thickness under moving milling force. Procedia CIRP, 58, 311 - 316 (2017)

4. Z. Cai, J. Geng, C. Zhang, Y. Du, Systematic solving of machining deformation and process optimization for complex thin-walled parts. Procedia CIRP, 56, 167 - 172 (2016)

5. W. Bai, X. Zhu, Finite element simulation and analysis of part deformation induced during milling of thin-walled aerospace monolithic structure parts. IEEE, Converence on Intelligent Computing and Intelligent Systems; 1, 440 - 444 (2010)

6. M. Estremsa, M. Arizmendia, W. E. Cumbicusa, A. Lópeza, Measurement of clamping forces in a 3 jaw chuck through an instrumented Aluminium ring. Procedia Engineering, 132, 456 - 463 (2015)

7. R. Thakore, T. Parsana, R. Dave, A review: Potential Failures in Grinding Process of Bearing Rings and its Solution. IJERT, 3, 666 - 670 (2014)

8. www.smwautoblok.com (Access: 30.01.2018)

9. www.schunk.com (Access: 30.01 .2018$)$ 